\title{
Fast Charging Scheduling under the Nonlinear Superposition Model with Adjustable Phases
}

\author{
ZHI MA and SHENG ZHANG, State Key Lab. for Novel Software Technology, Nanjing University \\ JIE WU, Temple University \\ ZHUZHONG QIAN, State Key Lab. for Novel Software Technology, Nanjing University \\ YANCHAO ZHAO, College of Computer Science and Technology, Nanjing University \\ of Aeronautics and Astronautics \\ SANGLU LU, State Key Lab. for Novel Software Technology, Nanjing University
}

\begin{abstract}
Wireless energy transfer has been widely studied in recent decades, with existing works mainly focused on maximizing network lifetime, optimizing charging efficiency, and optimizing charging quality. All these works use a charging model with the linear superposition, which may not be the most accurate. We apply a nonlinear superposition model, and we consider the Fast Charging Scheduling problem (FCS): Given multiple chargers and a group of sensors, how can the chargers be optimally scheduled over the time dimension so that the total charging time is minimized and each sensor has at least energy $E$ ? We prove that FCS is NP-complete and propose a 2-approximation algorithm to solve it in one-dimensional (1D) line. In a 2D plane, we first consider a special case of FCS, where the initial phases of all chargers are the same, and propose an algorithm to solve it, which has a bound. Then we propose an algorithm to solve FCS in a general 2D plane. Unlike other algorithms, our algorithm does not need to calculate the combined energy of every possible combination of chargers in advance, which greatly reduces the complexity. Extensive simulations demonstrate that the performance of our algorithm performs almost as good as the optimal algorithm.
\end{abstract}

CCS Concepts: • Networks $\rightarrow$ Wireless local area networks;

Additional Key Words and Phrases: Wireless charging, nonlinear superposition, efficient scheduling, interference-awareness

A preliminary version of this article was presented in Proceedings of the 15th IEEE International Conference on Mobile Ad-hoc and Sensor Systems (IEEE MASS 2018) (Ma et al. 2018a).

This work was supported in part by National Key R\&D Program of China (2017YFB1001801), NSFC (61872175, 61602238), NSF of Jiangsu Province (BK20181252, BK20160805), CCF-Tencent Open Fund, and Collaborative Innovation Center of Novel Software Technology and Industrialization. This research was supported in part by NSF grants CNS 1757533, CNS 1629746, CNS 1564128, CNS 1449860, CNS 1461932, CNS 1460971, and IIP 1439672.

Authors' addresses: Z. Ma and S. Zhang (corresponding author), State Key Lab. for Novel Software Technology, Nanjing University, 163 Xianlin Avenue, Nanjing 210023, China; emails: marszer@foxmail.com, sheng@nju.edu.cn; J. Wu, Department of Computer and Information Sciences, Temple University, SERC 362, 1925 N. 12th Street, Philadelphia, PA 19122; email: jiewu@temple.edu; Z. Qian, State Key Lab. for Novel Software Technology, Nanjing University, Nanjing 210023, China; email: qzz@nju.edu.cn; Y. Zhao, College of Computer Science and Technology, Nanjing University of Aeronautics and Astronautics, Jiangjun Rd. Campus: 29 Jiangjun Ave., Nanjing 211100, China; email: yczhao@nuaa.edu.cn; S. Lu, State Key Lab. for Novel Software Technology, Nanjing University, 163 Xianlin Avenue, Nanjing 210023, China; email: sanglu@nju.edu.cn.

Permission to make digital or hard copies of all or part of this work for personal or classroom use is granted without fee provided that copies are not made or distributed for profit or commercial advantage and that copies bear this notice and the full citation on the first page. Copyrights for components of this work owned by others than ACM must be honored. Abstracting with credit is permitted. To copy otherwise, or republish, to post on servers or to redistribute to lists, requires prior specific permission and/or a fee. Request permissions from permissions@acm.org.

(C) 2019 Association for Computing Machinery.

1550-4859/2019/09-ART48 \$15.00

https://doi.org/10.1145/3356342

ACM Transactions on Sensor Networks, Vol. 15, No. 4, Article 48. Publication date: September 2019. 


\section{ACM Reference format:}

Zhi Ma, Sheng Zhang, Jie Wu, Zhuzhong Qian, Yanchao Zhao, and Sanglu Lu. 2019. Fast Charging Scheduling under the Nonlinear Superposition Model with Adjustable Phases. ACM Trans. Sen. Netw. 15, 4, Article 48 (September 2019), 23 pages.

https://doi.org/10.1145/3356342

\section{INTRODUCTION}

Energy has always played an important role throughout human history. We human obtain wind power through wind turbines and obtain water power through hydro generators. With the development of society, the demand for energy is rasing. Not only has the demand for energy quantity increased, but also the demand for energy types has become more diverse. In past decades, Wireless Sensor Networks (WSN) have been proposed and been widely studied (Nishikawa et al. 2018; Noel et al. 2017; Jegadeesan and Venkatesan 2016; Li et al. 2014; Alphonsa and Ravi 2016). A WSN is a wireless network composed of a large number of static or mobile sensors in a self-organizing and multi-hop way, which can cooperatively sense, collect, process, and transmit the information of the perceived objects in the geographical area.

WSNs have many applications. Nishikawa et al. (2018) construct a WSN system that can sense and monitor the deformation of the ground caused by heavy rain in mountain areas with radio communication devices and sensor devices. WSNs are also used to monitor structural health (Noel et al. 2017), measure biological parameters in cattle farms (Jegadeesan and Venkatesan 2016), construct earthquake early warning systems (Alphonsa and Ravi 2016), and so on. However, sensors in WSNs are powered by small batteries, and constrained energy supply limits the lifetime of WSNs. Therefore, Energy Harvesting-based WSNs (EHWSNs) are proposed (Seah et al. 2009). In EHWSNs, sensors are replaced by energy harvesting sensors, which are designed to use various energy sources such as solar energy (Hsu et al. 2006; Jiang et al. 2005; Kansal et al. 2007; Raghunathan et al. 2005; Simjee and Chou 2006; Park and Chou 2006), active user power (Shenck and Paradiso 2001; Paradiso 2006), wind energy (Park and Chou 2006), and electromagnetic radiation energy (Niyato et al. 2007).

Although there are so many energy sources, Tong et al. (2010) pointed out that many energy sources may be limited by uncontrollable environmental conditions (e.g., cloudy skies, arid lands), and electromagnetic radiation is a cost-efficient way for sensors to harvest energy. In this article, wireless charging refers to the techniques that use electromagnetic radiation as the energy source.

Wireless charging techniques have been proposed to provide additional energy supply to prolong the lifetime of WSNs (Li et al. 2014; Zhang et al. 2018). Wireless energy charging has been regarded as a promising technology for prolonging sensor lifetime in wireless rechargeable sensor networks. With recent breakthroughs in wireless power transfer, it is possible to charge sensors over a long distance ( $>10 \mathrm{~m}$ away) with a fixed charger connecting to the power line.

However, long-distance charging brings a new problem: low transition efficiency, which means that the energy harvested by sensors is much lower than the energy sent by the chargers. As a result, it takes a much longer time to charge a EHWSN than expected. To accelerate long-distance charging, one way is to increase the chargers' power, but this may lead to electromagnetic radiation (EMR) pollution and harm humans (Gandhi et al. 2012). Another method is to add more chargers in EHWSNs and using multiple chargers to charge sensors at the same time, as the combined power energy will be stronger and charging time will be shortened. To calculate the combined charging power, almost all previous studies assumed that the combined energy from multiple chargers is additive (Dai et al. 2016; Deng-Peng 2009; Fréville 2004; Gao et al. 2015; Garcia et al. 1999; Peng et al. 2010), but this may not be the most accurate. Naderi et al. (2014a) points out that radio interference occurs when using multiple chargers to charge sensors at the same time. 


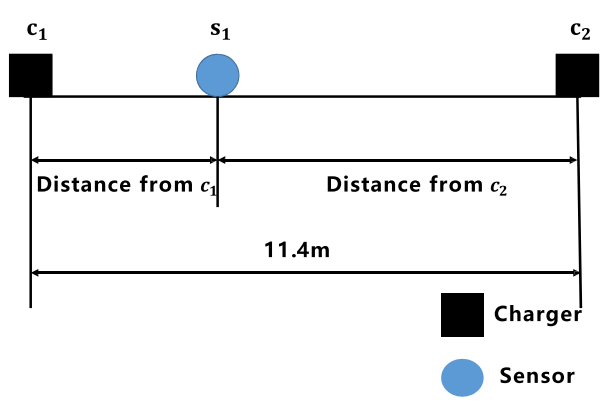

(a) The experimental scene.

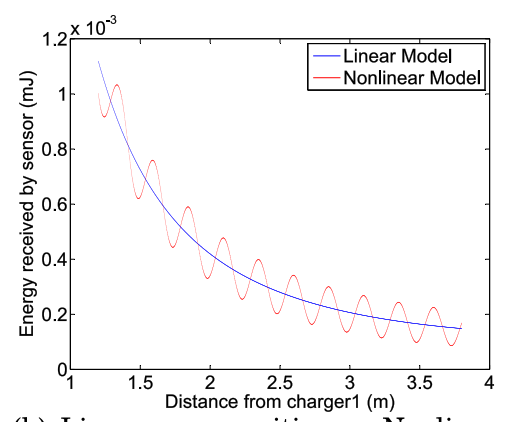

(b) Linear superposition vs Nonlinear superposition.

Fig. 1. Difference between linear superposition and nonlinear superposition. There are two chargers placed in a line, and they are $11.4 \mathrm{~m}$ apart from each other. There is a sensor moving from $c_{1}$ to $c_{2}$. The $x$-axis of the figure represents the distance between the sensor and $c_{1}$, and the $y$-axis represents the energy received by this sensor from those two chargers. The blue line in (b) represents the combined energy of two chargers using a linear superposition model, while the red line represents the combined energy using a nonlinear superposition model.

The difference between a linear superposition model and a nonlinear superposition model is shown in Figure 1. There are two chargers placed in a line, and they are $11.4 \mathrm{~m}$ apart from each other. There is a sensor moving from $c_{1}$ to $c_{2}$ along the line. The $x$-axis of Figure 1(b) represents the distance between the sensor and charger $c_{1}$, and the $y$-axis represents the received energy from those two chargers. The blue line in Figure 1(b) represents the combined energy of two chargers using linear superposition model, and the red line represents the combined energy using nonlinear superposition model. We can see from Figure 1(b) that when using a nonlinear superposition charging model, the combined energy of two chargers is not equal to the sum of their energy, as the chargers would strengthen each other in some places (which we call plus areas) but weaken each other in other places (which we call minus areas). Based on this, Guo et al. (2016) proposed a concurrent charging model and gave algorithms to solve the concurrent charging scheduling problem. Multiple chargers may weaken or strengthen each other depending on different distances; therefore, we cannot know the combined energy at a sensor node unless we explicitly know the set of working chargers and their positions and phases. Guo et al. (2016) calculates the charging energy of each charger set at each sensor node in advance, and algorithms were proposed based on these values. As a result of this preparatory process, the complexity of the proposed algorithm in Guo et al. (2016) grows exponentially with the number of chargers.

However, we observe that the nonlinear superposition model still has some properties. With these properties, we can reduce the computing complexity by no longer calculating the combined energy of each charger groups in advance.

This article focuses on the fast charging scheduling problem (FCS), which aims to schedule multiple chargers under the nonlinear superposition interference model without heavy calculations in advance. In our problem, a sensor can be charged by multiple chargers at the same time. Concurrent charging can accelerate the charging time, meanwhile maybe causing electromagnetic interference. To provide some intuitive insights into the structure of our problem, we first consider the scenario that all chargers and sensor nodes are distributed along a one-dimensional (1D) line. For this scenario, we propose a 2-approximation algorithm called FastPick. Then we investigate FCS in 2D EHWSNs. Because of the complexity in 2D EHWSNs, we first consider the condition that all initial phases of chargers are the same, and, after this, we try to solve the FCS in general 2D EHWSNs. 
Our main contributions are summarized as follows:

- We are the first to consider the FCS problem, which takes both ON/OFF decisions and heterogeneous initial phases into account, under the nonlinear superposition model. We theoretically prove that FCS is NP-complete.

- We propose a 2-approximation algorithm called FastPick to solve FCS in the 1D scenario.

-We propose the RoundPick algorithm to solve a special case of FCS in the 2D scenario and analyze its approximation. We also propose the WeightGreedyPick algorithm to solve FCS in general 2D EHWSNs.

- Simulations are conducted to evaluate the proposed solutions. The results are shown from different perspectives to provide conclusions.

The rest of the article are organized as follows. Section 2 surveys related works. Section 3 describes the concurrent charging model and formulates the problem. Section 4 analyzes the problem. Section 5 discusses the FCS in a one-dimensional line, and Section 6 solves FCS in a twodimensional plane. Section 7 includes the simulation results, and conclusions follow in Section 8.

\section{RELATED WORK}

Medium Access Control (MAC) protocols define rules for orderly access to the shared medium and play a crucial role in the efficient and fair sharing of scarce wireless bandwidth. Thus, in EHWSNs, MAC is an important technique that enables the successful operation of the sensors. In this section, we first introduce some backgrounds of MAC protocols, and then we introduce some related works about wireless charging.

\subsection{MAC Protocols for WSNs}

Sensor-MAC (S-MAC) (Ye et al. 2004) was a milestone protocol for WSNs. S-MAC defines a MAC protocol in which neighboring nodes form virtual clusters that share a common sleeping schedule. The time is divided into active and sleeping rounds. All the sensors of the cluster communicate in the active round, saving energy during the sleeping round. The active rounds are scheduled by periodical SYNC packets between the neighbors. S-MAC also identifies major sources of energy waste, including collision, overhearing, and idle listening. The S-MAC protocol focused on reducing energy consumption and is straightforward against the energy wasted in idle listening. Inspired by S-MAC, Dynamic Sensor MAC (DSMAC) introduces a dynamic duty cycle. DSMAC aims to decrease the latency for delay sensitive applications in battery-powered WSNs (Lin et al. 2004).

There are also some MAC protocols proposed for EHWSNs. Eu et al. studied several fundamental MAC layer approaches on EHWSNs (Eu et al. 2008). However, they assumed that the sensor does not have energy constraints thus the sensor never sleeps. So their insightful conclusions cannot be applied in common sensor networks. Fafoutis et al. proposed ODMAC, an on-demand MAC protocol for EHWSNs that is designed to minimize the energy wastage through idle listening (Fafoutis and Dragoni 2011). However, the energy harvesting rate is considered a parameter and the authors did not explain how it was generated.

Naderi et al. (2014b) proposed a new protocol called RF-MAC to work with RF energy harvesting. The RF-MAC protocol allows a sensor to broadcast its request for the energy packet containing its ID and then waits to hear from the wireless chargers in the neighborhood. However, in RF-MAC, a sensor node is charged only when it has communication request, which always incurs certain delay to execute the communication. 


\subsection{Wireless Charging in EHWSNs}

After talking about MAC protocols, we focus on wireless charging for EHWSNs with fixed chargers.

He et al. (2013) proposed how to deploy readers in a network to ensure that the WISP tags can harvest sufficient energy for continuous operation. They investigated the energy provision problem of finding the minimum number of RFID readers to cover a given EHWSN, and they showed that their algorithm can greatly reduce the number of readers compared with those assuming traditional coverage models. Pang et al. (2014) investigated the minimum charging coverage problem, which aims to recharge a set of sensors in a given area with the minimum number of wireless chargers. They introduced a partition algorithm to address this charging coverage challenge, and through theoretical analysis, they proved that the proposed algorithm can develop a solution close to the optimal one with guarantees approximation ratio. Dai et al. $(2012,2014)$ also focused on the charger location problem but took safety into account. They proposed PESA, a wireless charger Placement scheme that guarantees EMR safety for every location on the plane. Their experimental results showed that in terms of charging utility, their algorithm outperforms the prior form by up to $45.7 \%$. Zhang et al. (2018) considered wireless charging service provision in a two-dimensional target area and focused on optimizing charging quality, where the power of each charger is adjustable. The algorithms proposed by them perform very closely to the optimum and outperform the baseline algorithms.

Due to the low charging efficiency of fixed chargers, mobile chargers have been proposed. Mobile chargers are controlled to move around in EHWSNs and charge sensors nearby. Gao et al. (2015) proposed a new framework that can jointly schedule sensor activity and recharging to save the traveling energy of Recharging Vehicles (RVs). They proposed two schemes to manage sensor activity: balanced clustering and distributed sensor activation schemes. Based on schemes, they further introduced a new metric so that the energy demand in each cluster can be managed. Then they formulated the recharging problem into a Traveling Salesman Problem with Profits and proposed two algorithms to reduce travelling distance. The experiments results show that their algorithms can save travelling distances of RVs by $41 \%$ and $16 \%$, respectively. Zhang et al. (2015) proposed a scheduling algorithm called Pushwait to cover a one-dimensional EHWSN of infinite length, and they proved that Pushwait is the optimal algorithm in a 1D scenario. Li et al. (2014) proposed J-RoC-a practical and efficient Joint Routing and Charging scheme. They used proactive to guide the routing activities in the network and deliver energy to where it is needed. Evaluation results demonstrated that J-RoC significantly elongates the network lifetime compared to existing wireless charging based schemes. Sangare et al. (2017) developed a hardware platform using offthe-shelf radio frequency energy transfer hardware equipments to evaluate the practical performance of EHWSNs powered by radio frequency energy transfer. Based on the developed platform, they established an empirical model and used the empirical model to jointly optimize path planning and mobile charge scheduling for wireless-powered sensor networks. Numerical results showed that their derived policy significantly improves the performance of EHWSNs in different practical scenarios. However, in many scenarios the mobile chargers may not move freely. Additionally, the energy supply of the mobile chargers themselves is also a bottleneck.

Besides, all the works above are based on an assumption that the power received by one device from multiple chargers is linear additive, but this assumption may not be the most accurate.

Naderi et al. (2014a) pointed out that radio interference occurs when multiple chargers are used to charge one device, even if all the chargers transfer energy with high power. Interference may result in higher or lower levels of energy cancellation. Guo et al. (2016) proposed three algorithms to solve the concurrent charging scheduling problem based on the nonlinear superposition charging 
model. To the best of our knowledge, it is the first time that the nonlinear superposition charging model has been used to solve the charger scheduling problem. However, their algorithms had to calculate all charger sets utilities in advance. The complexity would grow exponentially with the number of chargers, which motivates us to formulate the FCS problem and solve it without calculations beforehand.

We have already done some works in wireless charging area. We have already done some works in Ma et al. (2018a, 2018b), and these works gave us a deeper understanding of the nonlinear charging model.

\section{MODEL AND PROBLEM FORMULATION}

In this section, we first propose our models, including the network model, charging model, harvesting model, and communication model. Different from previous works, our charging model is nonlinear superposition. Then we use these models to define the FCS problem.

\subsection{Network Model}

We consider a set of $N$ stationary sensor nodes $S=\left\{s_{1}, s_{2}, \ldots, s_{n}\right\}$ distributed over a twodimensional area. The location of the $i$ th node $s_{i}$ is denoted as $\left(x_{i}, y_{i}\right)$, and each node consumes energy for sensing, data reception, and transmission. There are also $M$ chargers, defined as $C=\left\{c_{1}, c_{2}, \ldots, c_{m}\right\}$, distributed in this area. The location of the $j$ th charger is denoted as $\left(x_{j}, y_{j}\right)$. There is a set $\left\{d_{i j} \mid 1 \leq i \leq N, 1 \leq j \leq M\right\}$ of distance between $c_{i}$ and $s_{j}$.

\subsection{Charging Model and Harvesting Model}

As we know, chargers use electromagnetic waves to transmit energy. According to Guo et al. (2016) and Naderi et al. (2015), we suppose that the amplitude of the frequency component $\omega_{0}$ in the chargers' power spectral density (PSD) curve is $A_{0}$ and that the corresponding initial phase is $\varphi_{0}$. Therefore, the power density of each charger at $\omega_{0}$ is $p_{0}=\frac{A_{0}^{2}}{2}$. Since the charging powers from wireless chargers weaken nonlinearly with distance, we assume, for simplicity, that the power attenuation factor is 2 . The radio signal of the frequency component $\omega_{0}$ arriving at the sensor node $s_{j}$ from the charger $c_{i}$ is expressed as

$$
a_{i 0}(t)=\frac{A_{0}}{4 \pi d_{i j} / \lambda} \cos \left(\omega_{0} t+\varphi_{0}-2 \pi \frac{d_{i j}}{\lambda}\right) .
$$

Based on this, Guo et al. (2016) proposed that the compound radio signal of frequency component $\omega_{0}$ at $s_{j}$ from a group of chargers $C$ is

$$
A_{0}^{j}(t)=\sum_{c_{i} \in C} a_{i 0}(t)=\sum_{c_{i} \in C} \frac{A_{0}}{4 \pi d_{i j} / \lambda} \cos \left(\omega_{0} t+\varphi_{0}-2 \pi \frac{d_{i j}}{\lambda}\right) .
$$

Then, we get the power of compound radio signal at sensor $s_{j}$ from charger set $C$ as follows:

$$
P_{j \mid C}=\int \overline{\left[A_{0}^{j}(t)\right]^{2}} d_{\omega}=P \sum_{c_{i} \in C} \frac{1}{d_{i j}^{2}}+P \sum_{c_{i} \in C} \sum_{\substack{c_{m} \in C \\ c_{m} \neq c_{i}}} \frac{1}{d_{i j} d_{m j}} \cos \left(2 \pi \frac{d_{i j}-d_{m j}}{\lambda}\right),
$$

where $P=\int p_{i} d_{\omega}$ is the radio power of each charger.

From this equation, we can see the nonlinear superposition charging effect in the concurrent charging. The equation above considers only distance and assumes that all sensors' initial phases are the same. With an adjustable initial phase, we can still use the equation to calculate and just need to add a variable of initial phase $\omega_{i}$. 
Generally, energy harvesting can be divided into two architectures: The first is the HarvestUse architecture, where energy is harvested just-in-time for use. The second is Harvest-Store-Use architecture, where energy is harvested whenever possible and stored for future use (Sudevalayam and Kulkarni 2010). In this article, we only consider the Harvest-Store-Use architecture. Denote $\left.P_{j}^{G}\right|_{C}$ as the power that sensor $s_{j}$ get from a group of chargers $C$. We assume $\left.P_{j}^{G}\right|_{C}=\left.\alpha * P_{j}\right|_{C}$, where $\alpha(0<\alpha<1)$ is the transition coefficient. From the former research (He et al. 2013), we know that if the radio power is lower than a threshold, then a sensor is not able to receive any energy from this radio. Taking this into account, we present the harvesting model as follows:

$$
\left.e_{j}\right|_{C, t}= \begin{cases}0 & \text { if }\left.P_{j}\right|_{C}<\epsilon \\ \alpha t\left(\left.P_{j}\right|_{C}-\epsilon\right) & \text { otherwise }\end{cases}
$$

where $\left.e_{j}\right|_{C, t}$ denotes the energy that $s_{j}$ harvested from a set of chargers $C$ during time $t$, and $\epsilon$ is the threshold of the radio power.

Each sensor also has an electric capacity. We set this capacity as E. So the energy harvesting model can be expanded as follows:

$$
\left.e_{j}\right|_{C, t}= \begin{cases}0 & \text { if }\left.P_{j}\right|_{C}<\epsilon \\ 0 & \text { if }\left.P_{j}\right|_{C}>\epsilon \text { and } e_{j}^{\prime}>E, \\ \alpha t\left(\left.P_{j}\right|_{C}-\epsilon\right) & \text { otherwise }\end{cases}
$$

where $e_{j}^{\prime}$ denotes the energy $s_{j}$ already received.

\subsection{Communication Model}

In this subsection, we introduce our communication model and MAC protocol used in this article.

Naderi et al. (2014a) gave an experimental study on the concurrent data and wireless charging for sensor networks, confirming the large interference range. Hence, to avoid the significant interference from the chargers, sensor nodes have to make communications only when no charger is active in the area.

We use the time-division multiple access (TDMA) control to insulate the wireless charging and wireless communication. That is, sensors work in turn for being charging and communications. Figure 2 shows the TDMA scheduling for charging and communication of sensors. During each turn of charging, it is expected to fully charge the sensor as soon as possible. After being fully charged, the sensors are ready for potential communications in the communication round, and chargers are not allowed to be active at this time. When the sensor nodes tend to exhaust their energy, another round of charging starts. Due to the low efficiency of long-distance charging, the charging round may need a relatively long time. Although a sensor in low-duty EHWSNs may have few communication requests in the charging round, the possibility that the whole network has many communication requests in the charging is non-ignorable. So we partition the charging round into multiple charging periods with the same duration by some time reservations. We can see from Figure 2 that these time reservations are evenly spaced in the charging period for potential communications of sensors.

Compared to RF-MAC where sensors are charged on-demand, the TDMA protocol is easier to implement and is flexible. Moreover, using the RF-MAC protocol to communicate always causes communication delay, and this delay would be even higher when many sensors have requires at the same time (which is common is EHWSNs). Therefore, it is necessary to charge sensor nodes in advance with TDMA instead of a hasty and crowded charging on-demand with RF-MAC. 


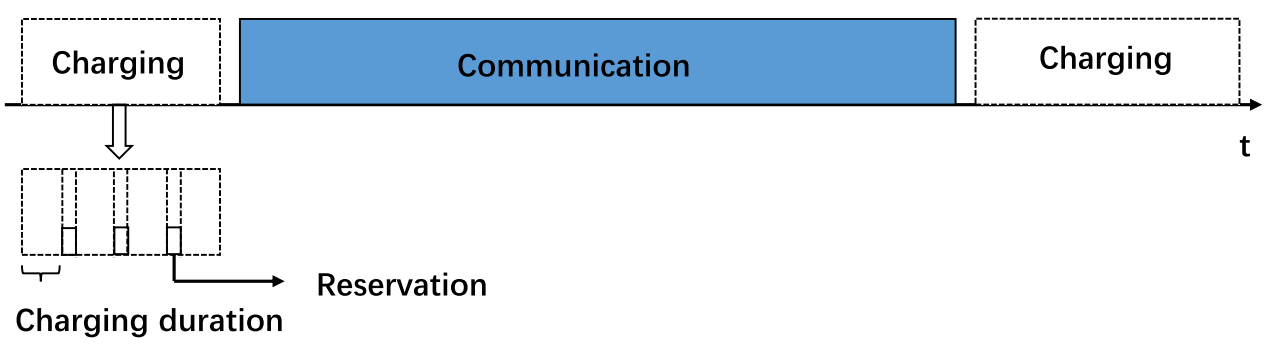

Fig. 2. TDMA scheduling for charging and communication of sensors.

\subsection{Problem Formulation}

In the above TDMA-MAC, the charging round is partitioned into multiple charging periods of equal length. To schedule the chargers, we need to ascertain the active time and inactive time of each charger in the charging periods. To simplify the scheduling instructions, we schedule each charger's active time and inactive time with the unit of charging period. We use $\Delta$ to represent the duration of each charging period, and we use the vector $H_{i}$ to denote the $i$ th charging schedule. For example, $H_{2}=[1,0,1,0]$ means that in the second charging period, the first and the third chargers are open while the second and the fourth chargers are closed. Also, we use the vector $\boldsymbol{X}_{\boldsymbol{i}}$ to denote the initial phases of chargers in the $i$ th charging period. For instance, $\boldsymbol{X}_{\mathbf{3}}=\left[\frac{\pi}{4}, 0, \frac{\pi}{16}, 0\right]$ means that in the third charging period, the initial phases of the second and the fourth charger are 0 , the initial phase of the first charger is $\frac{\pi}{4}$ and the initial phase of the third charger is $\frac{\pi}{16}$. Therefore, the main problem studied in this article is as follows:

Problem 1. Given a set $C$ of chargers with fixed position, a set $S$ of rechargeable sensors, a set $\left\{d_{i j} \mid 1 \leq i \leq N, 1 \leq j \leq M\right\}$ of distance between $c_{i}$ and $s_{j}$, and an energy capacity $E$ of each sensor, FCS is to find a set of multiple charging schedules $\left\{\mathrm{H}_{1}, \mathrm{H}_{2}, \ldots, \mathrm{H}_{\boldsymbol{k}}\right\}$ with corresponding initial phases of chargers $\left\{\boldsymbol{X}_{\mathbf{1}}, \boldsymbol{X}_{\mathbf{2}}, \ldots, \boldsymbol{X}_{\boldsymbol{k}}\right\}$, such that each sensor receives no less than $E$ energy, and $k$ is minimized.

\section{HARDNESS ANALYSIS}

In this section, we show that FCS is NP-complete.

THEOREM 4.1. The FCS is NP-complete.

Proof. We prove this by using the decision version of the FCS problem: Given a threshold $k$, does there exist a collection of charger sets $\left\{C_{1}, C_{2}, \ldots, C_{h}\right\}\left(C_{i} \subseteq C, i=1,2, \ldots, h\right)$ where $h$ is equal or less than $k$ ? In this problem, we assume that the initial phases of all chargers are the same.

We prove this decision problem by reduction from the knapsack problem (Martello et al. 2000), which is NP-hard. The decision version of the knapsack problem is as follows: Given a set of items $\mathbb{U}=\left\{e_{1}, e_{2}, \ldots, e_{m}\right\}$, each with a weight and a value, and an integer $k$, does there exist a collection of these items so that the total weight is less than or equal to the limit $W$ and the total value is $V$ ? Given an instance of the decision version of the knapsack problem, we construct an instance of FCS as follows:

-For each element $e_{j}$ in $\mathbb{U}$, we construct a charger set $C_{i}$ in FCS.

-For the weight of the item, we construct the charging period in FCS, and for the value of item, we construct the total energy harvested by the network in this period; we assume the length of all the charging periods are equal to $\Delta$.

-For the weight of knapsack, we use $k * \Delta$ to represent the limit. And for the given value $V$, we set $M * E$ as the value. 


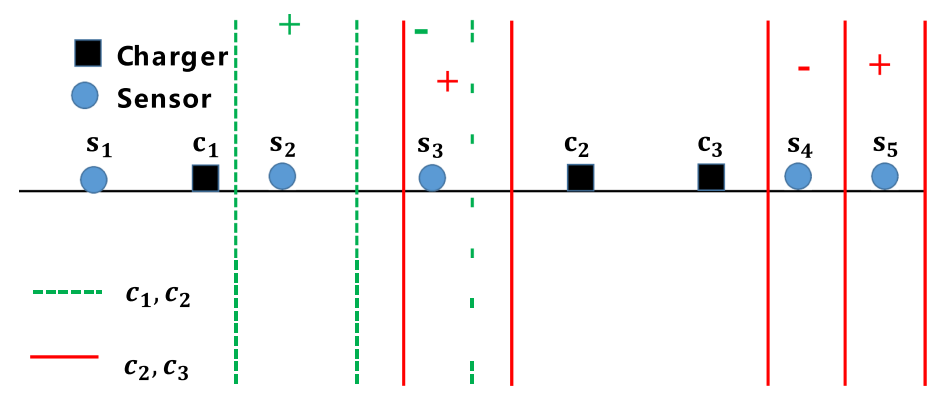

Fig. 3. One-dimensional plus areas and minus areas. Green lines represent the interactional areas of $c_{1}$ and $c_{2}$, and red lines represent the interactional areas of $c_{2}$ and $c_{3} . c_{1}$ and $c_{3}$ have no interactional area, because they are too far away from each other.

- After we pick a charger set $C_{i}$, we need to recalculate other periods' value, because while some sensors in this period may harvest energy, it is not sufficient to reach $E$. Therefore, we need to reduce other periods' value, as this complexity is the number of periods.

Combining these elements, we get the following special case of the decision version of the FCS problem: Given a limited time $k * \Delta$ and a period set, does there exist a collection of periods whose total size is less than or equal to $k$ so that all the sensors will harvest no less than $E$ energy (total is $M E)$ ?

The construction can be finished in polynomial time; thus, we reduce solving the NP-hard knapsack problem to a special case of FCS, implying that FCS is NP-hard.

\section{ONE-DIMENSIONAL LINE}

In this section, we discuss FCS in a one-dimensional line. We first show how initial phases influence charging, and then we propose an algorithm to solve FCS. After we give the complexity of our algorithm, we prove that our algorithm is 2-approximation.

\subsection{Rationale}

According to our observation, when the difference of phases between two chargers is less than $\lambda / 4$, these two chargers will strengthen each other. And when the difference is between $\lambda / 4$ and $\lambda / 2$, two chargers will weaken each other, where $\lambda$ represents the wavelength. As we can see from Figure 3, the initial phases of chargers $c_{1}$ and $c_{2}$ are the same, the green lines represent the interaction areas of $c_{1}$ and $c_{2}$, and sensor $s_{2}$ is distributed in their plus area, while $s_{3}$ lies in their minus area. If we increase the phase of $c_{1}$ by $\pi / 2$, then the total size of their plus areas and minus areas remain the same, but the positions will change: original plus areas become new minus areas and original minus areas become new plus areas. Generally speaking, in 1D line, once the distance and initial phase are determined, then the plus areas and minus areas are determined. When we change initial phases to different values, these areas will move along the line. Based on these observations, we propose FastPick, a 2-approximation algorithm, to solve the FCS in 1D line.

\subsection{The FastPick Algorithm}

FastPick is shown in Algorithm 1. Under the condition that the initial phases are adjustable, all chargers can be opened together, which means all the $\boldsymbol{H}_{\boldsymbol{i}}$ is $[1,1, \ldots, 1]$, and the initial phases of chargers are the only variables to be decided (line 1). First, we choose the sensor with the least energy (line 3). Next, we choose two chargers that are the closest to this sensor and adjust their 


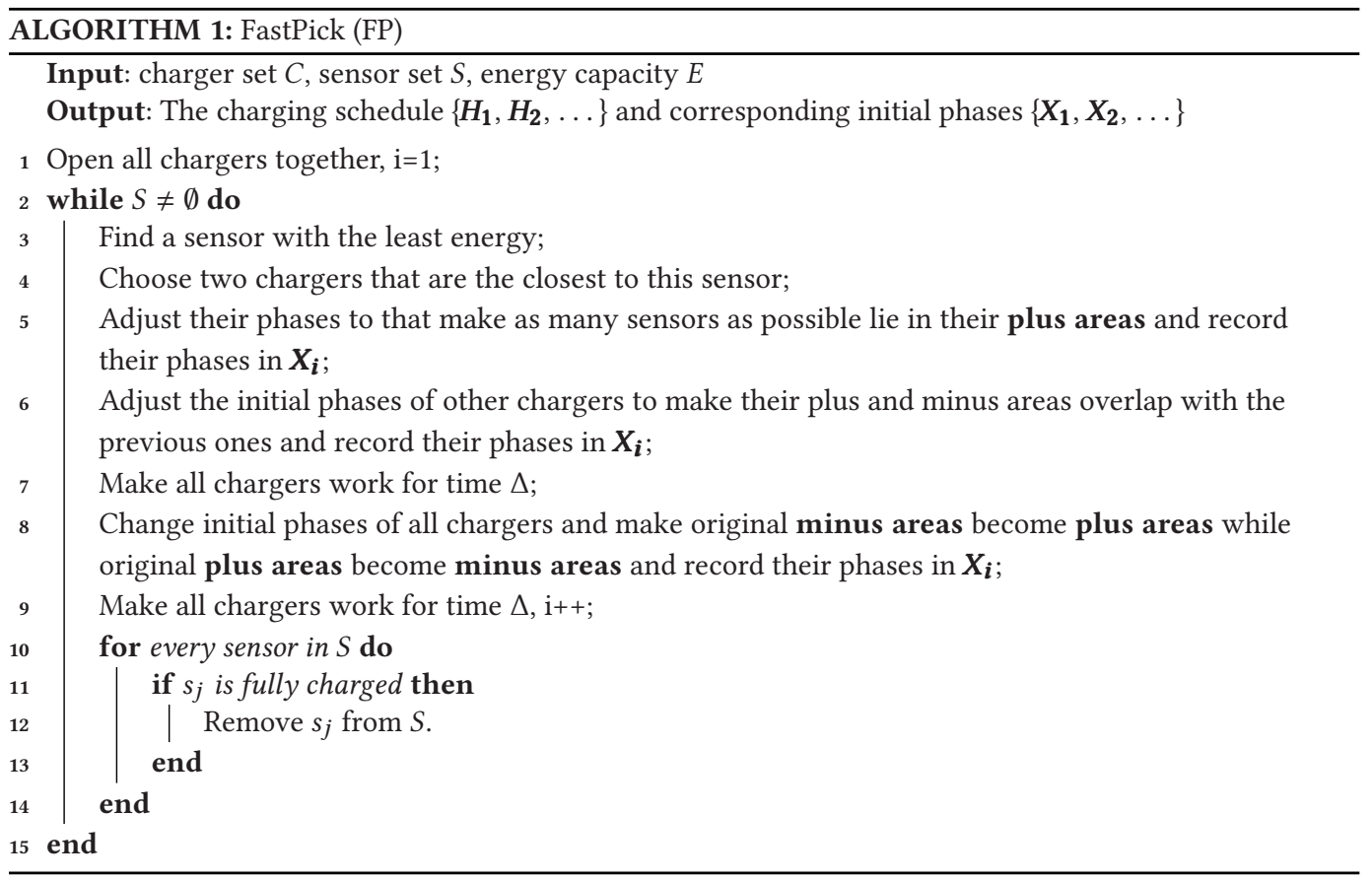

initial phases to make as many sensors as possible lie in their plus areas (lines 4 and 5). As we know that areas will move in parallel when initial phases change, we can make all chargers strengthen each other in the same places by adjusting their phases (line 6). In line 8 , we reverse the original minus areas and plus areas, make original minus areas become plus areas while plus areas become minus areas, this step ensures that our algorithm is 2-approximation. Algorithm 1 ends when all sensors are fully charged (line 2), and after each charging period, it will check whether a sensor is fully charged (lines 10-14).

\subsection{Approximation Ratio Analysis}

Now we show that FastPick is 2-approximation. First, we show a lower bound on the optimal charging time. Imagine that all chargers strengthen each other in all places (which cannot be achieved in reality); in this case, we can always open all chargers, and these chargers have no interferences. In doing so, we have a charging time $T_{a}$. Obviously, $T_{a}$ is a lower bound on the optimal charging time.

Next, we show that the charging time achieved by our algorithm FastPick is at most 2 times longer than $T_{a}$.

We know that sensors lie either in plus areas or minus areas. Sensors that lie in plus areas will get the most energy they can get from the chargers in this charging period. Sensors that lie in minus areas can hardly get energy in this period, but in the next period, minus areas and plus areas will reverse (line 8 in Algorithm 1), so these sensors will get the most energy in the next period. If we put these two periods together to construct a big period, then each sensor will get a little more energy in this big period than in the hypothetical situation that all chargers strengthen each other in all places. So the total charging time is $2 T_{a}-\epsilon$, which ensures $2 O P T-\epsilon$. Therefore, Algorithm 1 is 2-approximation. 


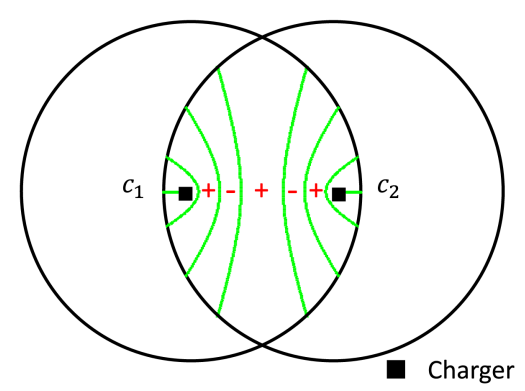

Fig. 4. Two-dimensional plus areas and minus areas. $c_{1}$ and $c_{2}$ represent chargers. "+" represents the plus areas of these two chargers, and "-" represents the minus areas.

\section{TWO-DIMENSIONAL PLANE}

In this section, we first propose an algorithm to solve a special case of FCS, where initial phases of all charger are the same. To distinguish it from the original FCS, we define this special case to be FCSF (FCS with Fixed phase). Then, we prove that under some conditions, our algorithm has a bound. After this, we propose the WeightGreedyPick algorithm to solve FCS in general 2D networks.

\subsection{FCSF Problem}

In the $1 \mathrm{D}$ line, the plus areas and minus areas are line segments, but in the $2 \mathrm{D}$ plane, the plus and minus areas are interspaces between some hyperbolas as shown in Figure 4. Given any pair of chargers, we can find their plus areas and minus areas, and use the area information to make our schedule.

To reduce calculation, we propose a method to partition the EHWSN. After partition, the whole EHWSN is divided into multiple grids, and we just need to focus on each grid independently. As we know, the coverage areas of chargers would coincide in some place; with the concurrent charging model, we cannot just look at one charger and leave other chargers irrespective.

\subsubsection{Partition. The partition should hold three conditions:}

CONDITION 1. Every sensor in one grid should be charged by chargers in this grid.

Condition 2. There is at least one charger in a grid.

Condition 3. The side length of the grid should be minimized, but no less than $2 * R$ ( $R$ is the charging radius).

After every charging period, we need to move the grid position by moving the grid toward the direction of the sensor with the least energy, and condition 3 guarantees that after moving, two chargers, which cause the overlap, will be in the same grid.

With these three conditions, we can find a bound of our algorithm. The worst case of the partition is that we can only get one grid, which means we do not make any partition. The best case of the partition is that every grid has exactly one charger.

6.1.2 The RoundPick Algorithm. RoundPick is showed in Algorithm 2. Under the condition that initial phases of all chargers are the same, charging schedule $\boldsymbol{H}_{\boldsymbol{i}}$ becomes the only variable to be decided. In lines 1-10, Algorithm 2 partitions the whole EHWSN. After partitioning, RoundPick circularly pick chargers during each iteration to decide each charging schedule (lines 11-19). The iteration terminates when all the sensors are fully charged. In each iteration, RoundPick first 

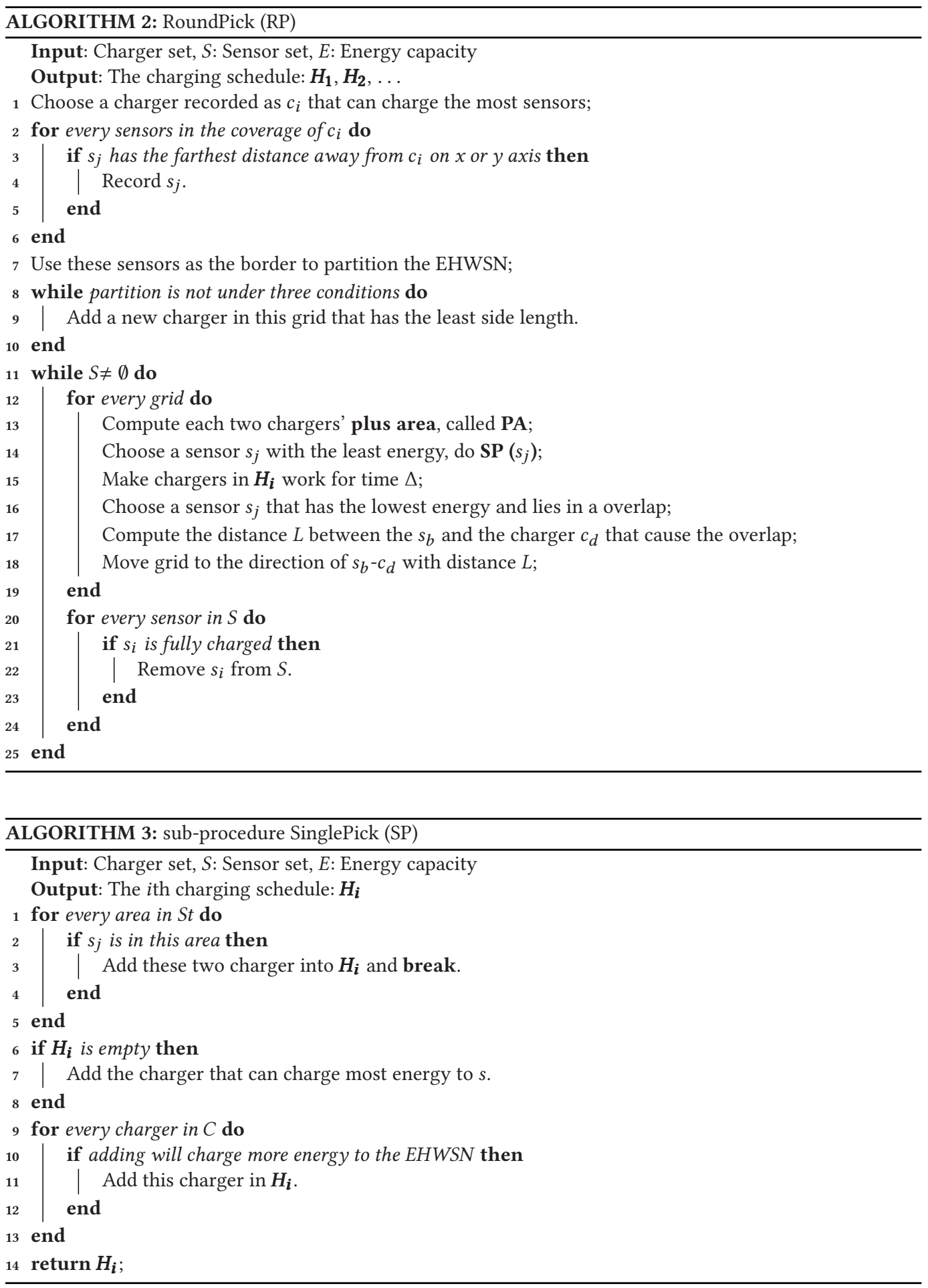


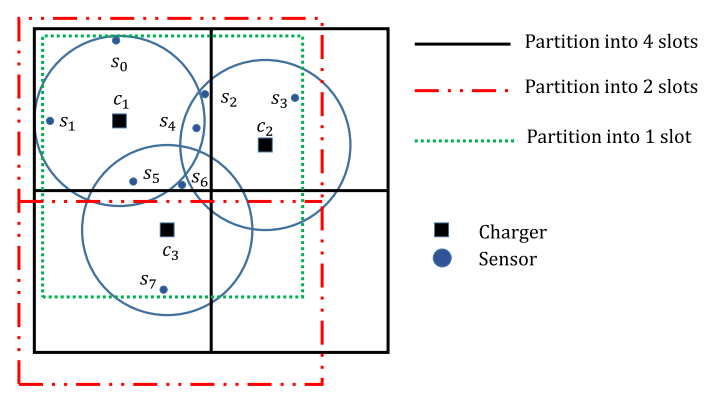

Fig. 5. An example of the partition of a WRAN. Black lines represent the partition that each grid has at most one charger. Red lines represent the partition that each grid has at most two chargers; green lines represent the partition that all chargers lie in the same grid.

computes each two chargers plus areas in each grid, called PA (line 13), and then the algorithm chooses a sensor with the least energy and uses Algorithm 2 to select chargers (line 14). Then, the chargers opening in this charging period are selected. After charging, we need to move the grid so that the sensors that overlap no longer overlap (lines 16-18). In this iteration, we still need to remove sensors with full energy, which means they already have $E$ energy (lines 20-25).

The complexity of Algorithm 2 is $\mathrm{O}\left(M^{3} N\right)$. The total number of the charging period is $\mathrm{O}(N)$, we can make this by increasing each charging period. The complexity to partition is $\mathrm{O}(M)$, because once we confirm a grid, others can be expanded, and the maximum number of chargers in a grid is $M$. During every charging period, the complexity of picking chargers is $\mathrm{O}\left(M^{3}\right)$; it is composed of two parts: the number of the grid and chargers in the grid. The most combination is $M^{3}$ mathematically. So the total complexity is $\mathrm{O}\left(M^{3} N\right)$.

6.1.3 Approximation Ratio Analysis. We take uniform distribution as an example and show the relation between bound and distribution. The hypothesis model is that all chargers strengthen each other to every sensor, so the schedule is to open all chargers together. This charging time is less than the optimal way. Under these three conditions, errors between neighbouring grids depend on the number of sensor sets in overlap areas. Suppose that sensors and chargers are uniformly distributed, then the most overlap areas of a grid are 4, and the sensors in these overlaps are half of sensors in this grid. If chargers in other grids will strengthen these overlaps, then we just ignore them. If chargers will weaken the overlaps, then the worst case is that half the sensors would get less energy. But after every period, we do the movement operation, which will make sensors in minus areas in a new grid without charging conflict with the other grid. But one operation of movement can only make one direction in the 2D plane conflict-free, which means that we should do another movement to make another direction become conflict-free. In every charging period, we first choose a pair of chargers that can charge the most energy, and then a new charger can be added if and only if this charger has the same plus areas with the first two chargers. So in every charging period, we can make sure that half of the sensors charge the most energy. Since half of them are influenced by other grids, eventually, only $1 / 4$ sensors get the most energy. The bound is $\alpha *\left(N_{d}+1\right)-\epsilon$, where $\alpha$ is the charging ability compared with OPT in each grid, $N_{d}$ is the influenced direction in grid $\left(N_{d}=\{1,2\}\right)$, and 1 represents the original grid, while $\epsilon$ is the error related to the actual charging condition. In uniform distribution, the upper bound is $(2-\epsilon) *(2+1)-\epsilon=6-4 \epsilon$.

6.1.4 Example in 2D. In this section, we give an example to show the running process of our algorithm. A simple example is shown in Figure 5, and Table 1 shows the actual charging energy. 
Table 1. The Receiving Energy of Sensors During One Time Period in a 2D Plane

\begin{tabular}{|c|c|c|c|c|c|c|c|c|}
\hline & $s_{0}$ & $s_{1}$ & $s_{2}$ & $s_{3}$ & $s_{4}$ & $s_{5}$ & $s_{6}$ & $s_{7}$ \\
\hline$c_{1}$ & 2 & 2 & 0 & 0 & 2 & 2 & 0 & 0 \\
\hline$c_{2}$ & 0 & 0 & 2 & 2 & 3 & 0 & 0 & 0 \\
\hline$c_{3}$ & 0 & 0 & 0 & 0 & 0 & 3 & 3 & 3 \\
\hline$c_{1}, c_{2}$ & 2 & 2 & 2 & 2 & 0 & 2 & 0 & 0 \\
\hline$c_{1}, c_{3}$ & 2 & 2 & 0 & 0 & 2 & 5 & 2 & 2 \\
\hline$c_{2}, c_{3}$ & 0 & 0 & 2 & 2 & 3 & 3 & 3 & 3 \\
\hline$c_{1}, c_{2}, c_{3}$ & 2 & 2 & 2 & 2 & 0 & 5 & 2 & 2 \\
\hline
\end{tabular}

Suppose the energy capacity of each node is $E=10$. According to algorithm, we first partition the EHWSN and find the charger that can charge the most sensors. So we choose $c_{1}$ and use it as the division criterion to partition the whole EHWSN. As a result, we get four grids, as shown in Figure 5, with black lines. Afterward, we check whether this partition follows our three conditions. Obviously, $s_{2}$ belongs to grid 1 , but since it is not charged by $c_{1}$, this partition is thus incorrect. Then, we should add one charger; without loss of generality, we add $c_{2}$, and we use the new side length of the grid to partition the EHWSN, which is shown in Figure 5 with red lines. After partition, we get two grids, and we check it again. $s_{6}$ lies in grid 1 but is not covered by $c_{1}$ and $c_{2}$, which means this partition is wrong, so we add $c_{3}$ and make the whole EHWSN a grid, which is shown in Figure 5 with green lines. This is the worst case, because we do not actually partition the EHWSN. After partitioning, Algorithm 2 selects a sensor with the least energy; in the first iteration, all sensors have energy 0 . Without loss of generality, we choose $s_{0}$ and add $c_{1}$ in $\boldsymbol{H}_{\mathbf{1}}$. According to Algorithm 2, we add $c_{3}$ into $H_{1}$. That is the first charging period schedule. After this charging period, the sensors energy are $\{2,2,0,0,4,5,3,3\}$. In the second iteration, we select $s_{2}$ and choose $c_{2}$ into $H_{2}$; then, we add $c_{3}$ into $\boldsymbol{H}_{2}$. After this charging period, the sensors energy are $\{2,2,2,2,8,8,6,6\}$. In the third period, we choose $c_{1}, c_{3}$. Because $s_{4}$ and $s_{5}$ have an energy of 8 now, the energy harvested by them is at most 2 due to our harvest model in Section III. So we can add $c_{2}$ into $H_{3}$, too. After this charging period, the sensors energy are $\{4,4,4,4,8,10,9,9\}$. In the fourth iteration, we choose $c_{1}, c_{2}, c_{3}$, and the sensors energy after charging are $\{6,6,6,6,8,10,10,10\}$. In the fifth period, we choose $c_{1}, c_{2}$, and the sensors energy are $\{8,8,8,8,8,10,10,10\}$. In the sixth period, we choose $c_{1}, c_{2}$, and the sensors energy after charging are $\{10,10,10,10,8,10,10,10\}$. In the seventh period, we choose $c_{1}$ only. Therefore, the total charging periods of our algorithm is 7 .

\subsection{FCS in Two-Dimensional Plane}

In the previous section, algorithm RoundPick solves FCSF, where the initial phases of all chargers are the same. In this subsection, we discuss FCS in a two-dimensional plane. We first show the difficulty in handling FCS in the 2D plane, and then we propose an algorithm to solve FCS. Then we analyze the complexity of our algorithm.

In Section 4, we prove that FCS is NP-complete under the condition that initial phases of all chargers are the same, which means FCSF is NP-complete. With adjustable initial phases, solving a FCS problem means solving a large number of FCSF problems. Once we change the phase of any charger, the input of our original FCSF problem changes, so the original FCSF problem changes into a new FCSF problem, thus making it hard to find a solution for a FCS problem.

We propose the WeightGreedyPick (WP) algorithm to solve the FCS in a 2D plane. Our goal is to use the minimal time to charge each sensor with at least $E$ energy; therefore, the energy charged in each period should be maximized. So the amount of energy received by the whole network should 


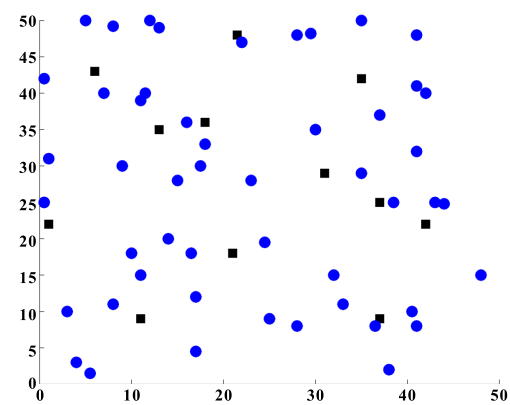

(a) The Placement with $N=12, M=50$.

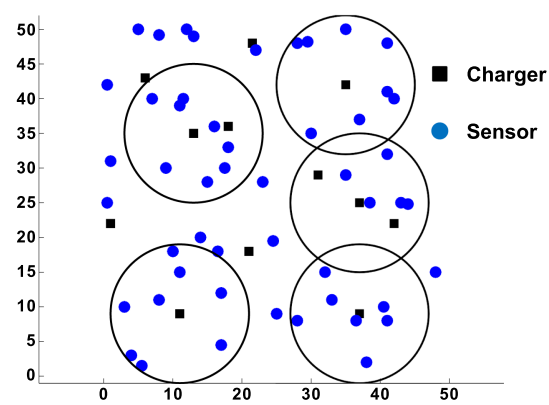

(b) A MCS of a EHWSN with $N=12, M=50$.

Fig. 6. A EHWSN with $N=12, M=50$.

be considered. Meanwhile, some sensors can be charged by only one or two chargers; therefore, the energy received by a single sensor should be considered. So the number of chargers that are able to charge a sensor should be taken into account when designing an algorithm. In this article, we only discuss instances where the length of periods are the same.

To balance the received energy by a single sensor and the received energy by the whole network, we give each sensor a weight $w$, which is represented as $E^{\prime} / r$, where $E^{\prime}$ is the energy remained to be charged, and $r$ is the number of chargers that can charge this sensor. We also give each charger a weight $w^{\prime}$, which is the sum of the weights of the sensors that can be charged by this charger $\left(w_{i}^{\prime}=\sum_{s_{j}} w_{j}\right.$, where $s_{j}$ is the sensor charged by $\left.c_{i}\right)$. We use $W$ to represent the set of the weight of the chargers.

The main idea of our algorithm is as follows: We find a maximum unique covering set (MCS) first and then expand this set by adding more chargers. Maximum unique coverage means every item can be covered at most once, and the total weight of the set is maximized. As we know, MCS is an NP-complete problem (Demaine et al. 2008) and has no polynomial way to solve it. We propose a weight-greedy way to find a MCS. We give each charger a weight defined above. Every time we pick or add a charger, we pick or add the charger with the maximum weight.

We show why using the weight we designed to select charger can balance both individual and overall energy. Supposing sensor $s_{i}$ can be charged only by one charger named $c_{k}$, while $s_{j}$ can be charged by four chargers, and $c_{k}$ weakens other chargers. It is obvious that $c_{k}$ should be opened for $E / p\left(c_{k}\right)$ time so that $s_{j}$ can be fully charged, where $p\left(c_{k}\right)$ is the power received by sensor $s_{j}$ from charger $c_{k}$. By the weight of sensors as $E^{\prime} / r, s_{j}$ 's weight is divided into four parts while $s_{i}$ 's weight remains the same. So the priority to select $c_{k}$ is increased. After we select a charger, we need to remove covered sensors from the universal set and also remove chargers that can charger these covered sensors. As a result, every sensor is covered by at most one charger. Then we need to calculate every charger's weight again. The complexity to find an MCS is $\mathrm{O}(M N)$. Figure 6 shows an example of an MCS.

Here we set the initial phases of all chargers to 0 , and only use a step value of phase $\delta$ to adjust. This is reasonable, because the interference areas are only affected by the D-value of two chargers. We can change the $\mathrm{D}$-value of the chargers by adding $\delta$ to some chargers.

In Algorithm WeightGreedyPick, we first initialize $W$ as a set of all chargers weights in line 1. WeightGreedyPick iteratively decides each charging schedule (lines 2 to 11). The iteration terminates when all the sensors are fully charged $(S \neq \emptyset$ line 2$)$. In each iteration, WeightGreedyPick first finds a MCS and then adds chargers into $H_{i}$ during this charging period. After this, we set all $w^{\prime}$ to 0 and recalculate the weight of chargers that has not been picked. When $\max \left\{w^{\prime} \in W\right\}>0$, we add this charger into $\boldsymbol{H}_{\boldsymbol{i}}$. Then we adjust the phase of this charger according to $\delta$ and choose 


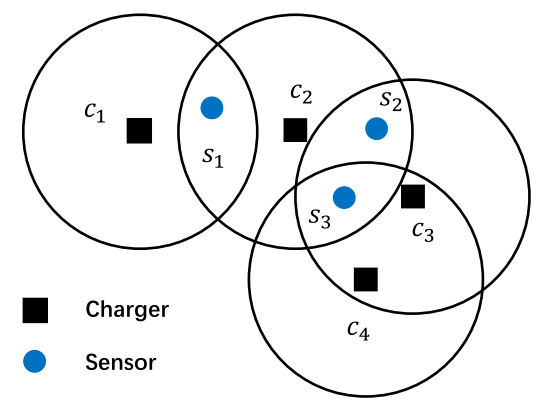

Fig. 7. Example of the placement of four chargers and three sensor nodes.

Table 2. Example of the Charging Utilities of Four Charger Sets at Three Sensor Nodes

\begin{tabular}{|c|c|c|c|}
\hline & $s_{1}$ & $s_{2}$ & $s_{3}$ \\
\hline$c_{1}$ & 3 & 0 & 0 \\
\hline$c_{2}$ & 2 & 3 & 2 \\
\hline$c_{3}$ & 0 & 3 & 3 \\
\hline$c_{4}$ & 0 & 0 & 2 \\
\hline$c_{1}, c_{2}$ & $3\left(\varphi_{1}=\varphi_{2}=0\right) / 4\left(\varphi_{1}=0, \varphi_{2}=\frac{\pi}{2}\right)$ & 3 & $1\left(\varphi_{2}=\varphi_{3}=0\right) / 4\left(\varphi_{2}=0, \varphi_{3}=\frac{\pi}{2}\right)$ \\
\hline$c_{2}, c_{3}$ & 2 & $0\left(\varphi_{2}=\varphi_{3}=0\right) / 5\left(\varphi_{2}=0, \varphi_{3}=\frac{\pi}{2}\right)$ & $0\left(\varphi_{2}=\varphi_{4}=0\right) / 3\left(\varphi_{2}=0, \varphi_{4}=\frac{\pi}{2}\right)$ \\
\hline$c_{2}, c_{4}$ & 2 & 3 & $5\left(\varphi_{3}=\varphi_{4}=0\right) / 1\left(\varphi_{3}=0, \varphi_{4}=\frac{\pi}{2}\right)$ \\
\hline$c_{3}, c_{4}$ & 0 & 3 & $4\left(\varphi_{2}=\varphi_{3}=\varphi_{4}=0\right) / 5\left(\varphi_{2}=0, \varphi_{3}=\varphi_{4}=\frac{\pi}{2}\right)$ \\
\hline$c_{2}, c_{3}, c_{4}$ & $2\left(\varphi_{1}=\varphi_{2}=0\right) / 2\left(\varphi_{1}=0, \varphi_{2}=\frac{\pi}{2}\right)$ & $0\left(\varphi_{2}=\varphi_{3}=0\right) / 5\left(\varphi_{2}=0, \varphi_{3}=\frac{\pi}{2}\right)$ & \multicolumn{2}{|c|}{} \\
\hline
\end{tabular}

the phase that can make the energy received by the EHWSN as big as possible. If the energy received by the whole EHWSN becomes smaller no matter how we change the phase of the new adding charger, then this charger will be removed. After adding a charger, WeightGreedyPick recalculates $W$ because of the charging interference. This operation continues until no chargers can be added, which means adding any charger would have negative effect on the whole network (lines 8-10). WeightGreedyPick makes the chargers in $\boldsymbol{H}_{\boldsymbol{i}}$ charge for $\Delta$ time (line 12). After each charging period, the algorithm removes sensors that are fully charged from $S$ (lines 13 to 17). Finally, we recalculate $W$ and add 1 to $i$ (line 18).

\subsection{Example and Analysis}

In this subsection, a simple example of the algorithm is shown in Figure 7, and Table 2 shows the actual charging energy with different initial phases of chargers. For simplicity, every charger has only two different phases to choose. Suppose the energy capacity of each node $E=10$.

According to Algorithm 4, we first find a MCS, which is $c_{2}$, then we add other chargers if the total energy harvested by the network increases after adding this charger. So we add $c_{1}$ and adjust the phase of $c_{1}$ to be 0 , and the phase of $c_{2}$ to be $\frac{\pi}{2}$, and then this iteration ends. After charging, the current energy harvested in the node is $\{5,3,2\}$. In the next period, we first check whether any sensor is fully charged. Then, we continue this operation. Obviously, $c_{1}$ and $c_{2}$ will be picked in the next two periods. The current energy of each sensor increases to be $\{10,9,6\}$. Now sensor $s_{1}$ is fully charged, and we remove it and find MCS again, which is $c_{2}$ or $c_{3}$. Suppose we choose $c_{3}$; then $c_{4}$ and $c_{2}$ will be added. Then we adjust the phase of $c_{2}$ to be 0 , the phases of $c_{3}$ and $c_{4}$ to be $\frac{\pi}{2}$. After this charging period, all sensors are fully charged. The total charging period is 4 . 


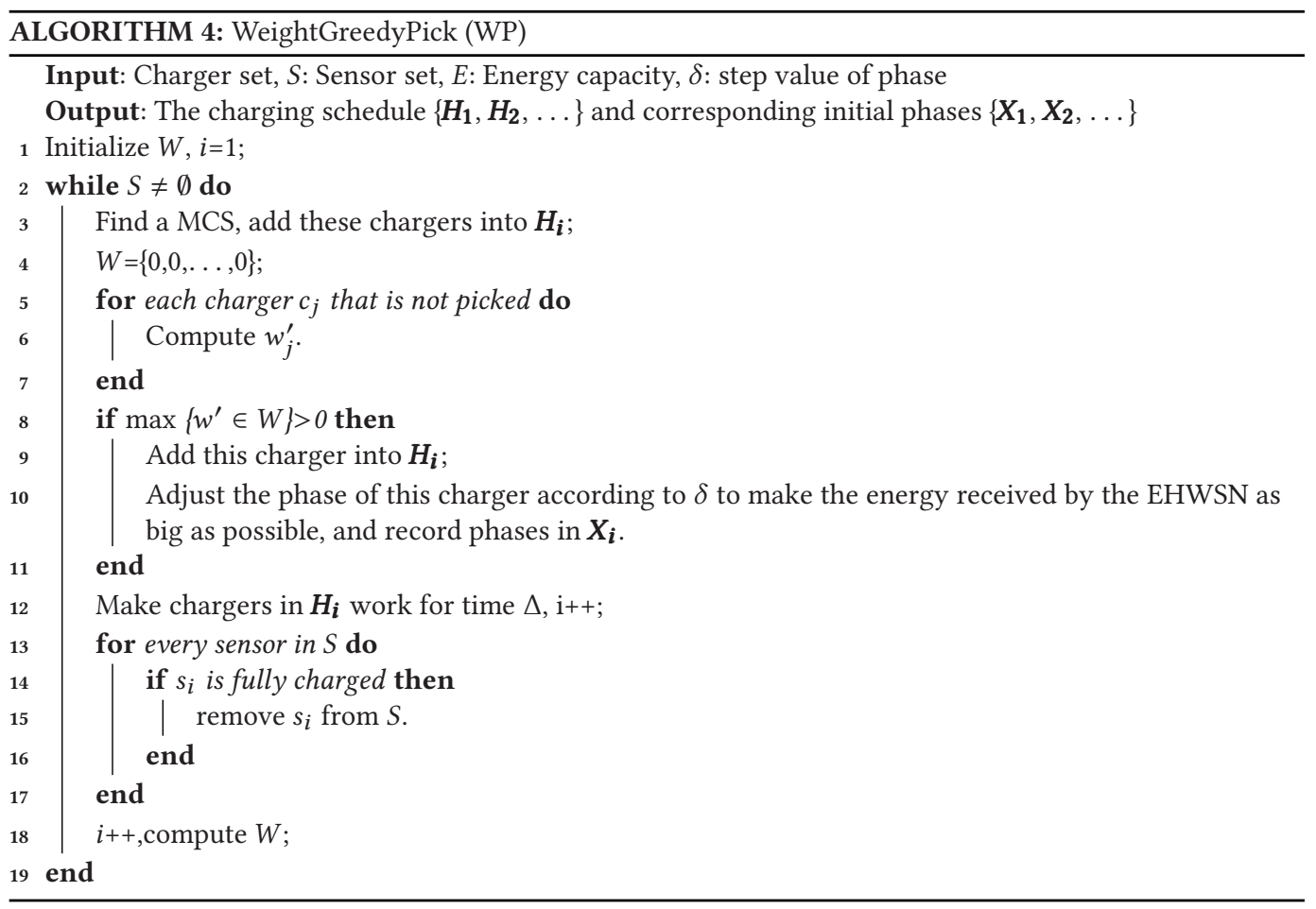

Our algorithm would obtain the optimal solution in two extreme cases. The first is when all chargers strengthen each other. Obviously, the optimal method is to turn on all the chargers, which is the same answer that our algorithm would give. The other case is when all chargers weaken each other. In this case, we should make the interference the smallest possible in each period. The answer is to turn on chargers in the MCS, which is also the same answer that our algorithm gives.

The complexity of this algorithm is $\mathrm{O}\left(M^{2} N^{2}\right)$. The while-loop runs at most $\mathrm{O}(M)$ iterations, and we can make this by increasing the charging time of each period. In each iteration, while-loop runs at most $N$ times and for-loop runs at most $\mathrm{O}(N)$ times, because the complexity of calculating the weight $w^{\prime}$ of one charger is $\mathrm{O}(M N)$. The complexity of finding a MCS is $\mathrm{O}(M N)$. The total complexity is $\mathrm{O}\left(M^{2} N^{2}\right)$.

\subsection{Summary}

In consideration of the difficulty in solving FCS in a 2D plane, we first discuss a special case of FCS, which we call the FCSF problem. We propose the RoundPick algorithm to solve FCSF and obtain a bound of $6-4 \epsilon$ when sensors and chargers are uniformly distributed. After giving an example of RoundPick, we try to solve FCS in a 2D plane. We propose the WeightGreedyPick algorithm to solve it.

\section{EXPERIMENTS}

In this section, we conduct a series of simulations with a Matlab tool to evaluate the performance of the proposed algorithm, including RoundPick for FCSF and WeightGreedyPick for FCS. 


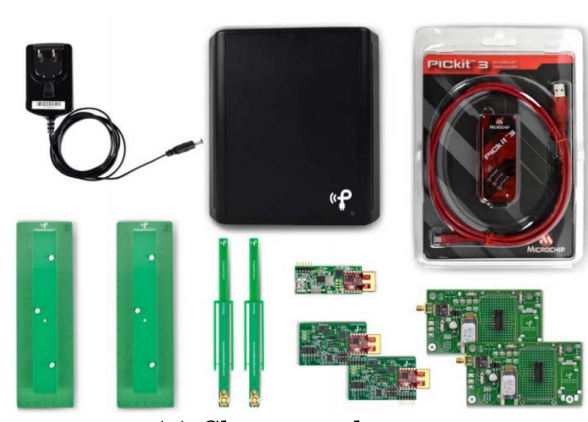

(a) Charger and sensor.

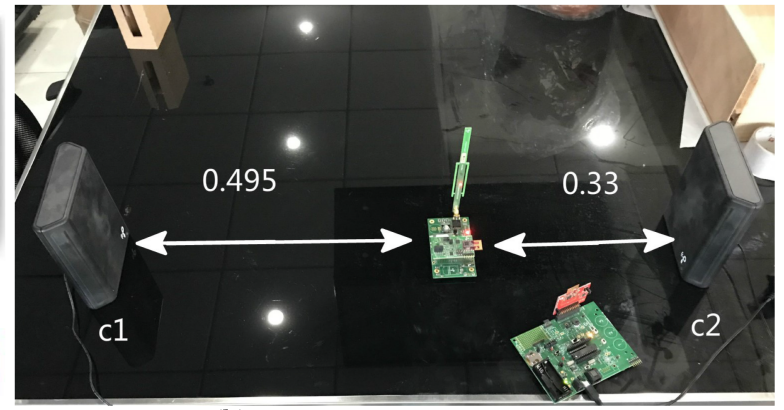

(b) experiment environment.

Fig. 8. Experiment devices.

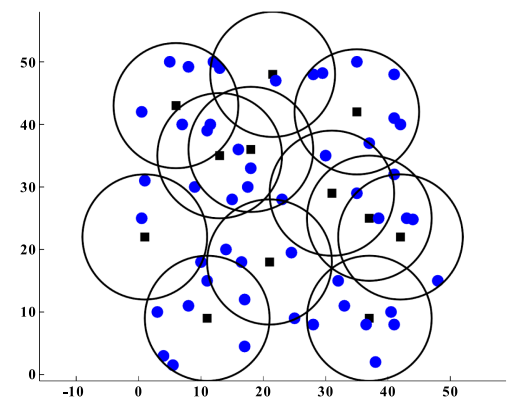

(a) The placement with 12 chargers and 50 sensors.

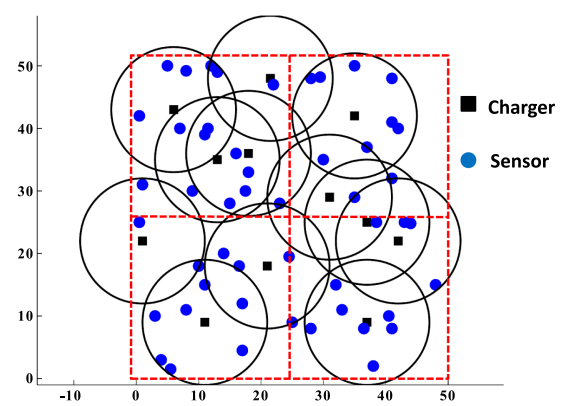

(b) A partition of a EHWSN with 12 chargers and 50 sensors.

Fig. 9. An example of the partition.

\subsection{Experimental Settings}

We assume wireless devices and chargers are randomly distributed over a $50 \mathrm{~m} \times 50 \mathrm{~m}$ area. In the simulations, we employed the energy harvesting model present in Section 3. For the deployments and the harvesting model, the time is calculated, and the procedures for the proposed algorithm are executed in Matlab. Figure 8 shows our experiment equipments. We use the powercast TX91501$915 \mathrm{MHz}$ to transmit energy. Moreover, we set the charger's power to be $915 \mathrm{MHz}$, which makes the wavelength $\lambda=0.33 \mathrm{~m}$. We set the threshold of harvesting power as $\epsilon=15 \mu \mathrm{W}$, transition efficiency as $\alpha=0.25$, and each charging period as $\Delta=20 \mathrm{~s}$.

Based on these parameters, we calculate the distance threshold, $0.25 * 4 /\left(4 \pi^{*} d\right)^{2}=0.015 \mathrm{~mW}$. Then we calculate that $d \approx 6.78 \mathrm{~m}$, which means that when the distance between a sensor and a charger is over $6.78 \mathrm{~m}$, the sensor will harvest no energy from that charger. In this simulation, the default number of chargers is $N=12$. The default number of sensors is $M=50$, and the default energy capacity is $E=4 \mathrm{~mJ}$. Figure 9(a) gives an example of the default placement. Figure 9(b) illustrates the partition that RoundPick finds in the first iteration.

\subsection{Baseline Setup}

(i) Baselines of RoundPick for FCSF are as follows: Currently there is only one algorithm available for FCSF with an actual charging model. The algorithms proposed in Guo et al. (2016) calculate all the charger groups' charging abilities in advance, and the performance of the Genetic Algorithm (GA) they proposed is almost as good as the brute-force algorithm. We consider the GA as the optimal algorithm (OPT). 
We also introduce a Random Algorithm (RA) for comparison. In addition, we compare our algorithms with the random algorithm that consists of two phases. The first phase is removing $k$ chargers, which cannot charge any sensor, because some sensors may be fully charged after some periods from $C$. The second phase is to randomly select $\beta(N-k)(0<\beta<1)$ chargers in each period. In this simulation, we set $\beta$ to be 0.8 .

(ii) Baseline of WeightGreedyPick for FCS is as follows: Since we are the first to consider adjustable initial phases alone with a nonlinear superposition charging model in a wireless charging field. There is no other algorithm available to contrast WeightGreedyPick. In fact, FCS is too hard to given an approximation algorithm. Here we introduce the RandomRoundPick (RRP) algorithm for comparison. Given a set of fixed initial phases, RoundPick is able to output a charging schedule. So we randomly set the initial phases of chargers as the input of RoundPick and use the minimum value of several random inputs as the output to contrast the output of WeightGreedyPick.

For WeightGreedyPick, we set the $\delta$, the step value of the phase, to be $\frac{\pi}{16}$. And we set the initial phases of all chargers to be 0 .

\subsection{Evaluation Results}

In general, RoundPick achieves a near-optimal solution and outperforms the random algorithm.

In Figure 10(a), the number of charging periods decreases as the number of chargers grows. This is obvious, because with more chargers, more sufficient energy will be supplied in the same period, thereby reducing the charging time. According to the algorithms, the performance of RoundPick is close to OPT when the number of chargers is small, and as the number of chargers increases, the operation performance of RoundPick is gradually withdrawn by OPT. This is because when the number of chargers is small, the electromagnetic interference will be limited and will tend to select all the chargers.

In Figure 10(b), the total charging time grows with the growth in the number of sensors, and there is a very good explanation, because with more sensors, interference will become more common, so the total charging length is increased. From each of the algorithms, the performance of RoundPick is close to OPT when the number of sensors is small, and as the number of sensors increases, the performance of RoundPick is worse than OPT. This is because when the number of sensors is small, the electromagnetic interference caused by the sensor will be small, and the overall charging power of the network will be higher than that of a single sensor.

In Figure 10(c), when the energy capacity goes up, the total charging time grows with the growth of the capacitance, obviously, because the total harvesting grows, thereby increasing the charging time. This is due to the fact that the electromagnetic interference is not large in the case of an hour of electrical capacity, but as the capacitance increases, the influence of electromagnetic interference on the charging will be amplified.

In Figure 10(a) and Figure 10(b), when the number of sensors or chargers decreases, the chance of charging interferences goes down. As a result, the number of charging periods our algorithm calculates is close to the optimal one. However, when the number of sensors or chargers goes up, the performance of our algorithm is bad, because the interference becomes more common and is hard to control. The only way to make the best choice is to calculate each group of chargers when interference occurs.

As for the time complexity, it can be seen from Figure 10(d)-(f) that the running times of the RoundPick and RA are much lower than those of the optimal algorithm. As we mentioned before, the complexity of the OPT algorithm grows exponentially with the number of chargers. The running times of these three algorithms all increase with the charger scale, sensor scale, and energy capacity, which is as expected. 


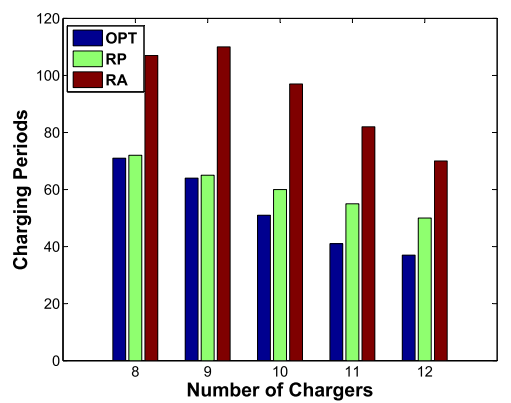

(a) Charging periods vs Number of chargers.

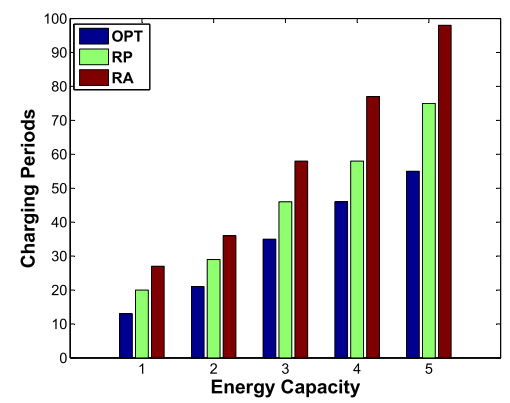

(c) Charging periods vs Energy capacity.

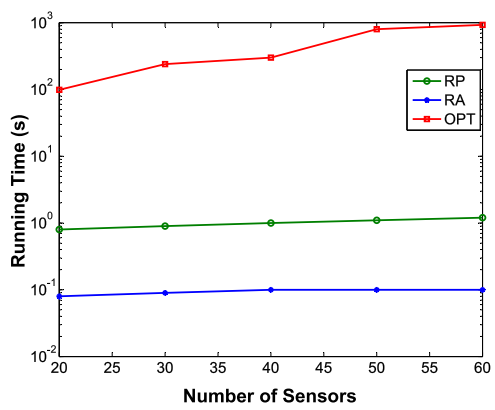

(e) Running time vs Number of sensors.

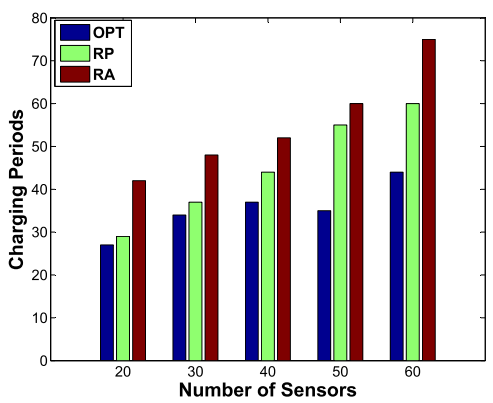

(b) Charging periods vs Number of sensors.

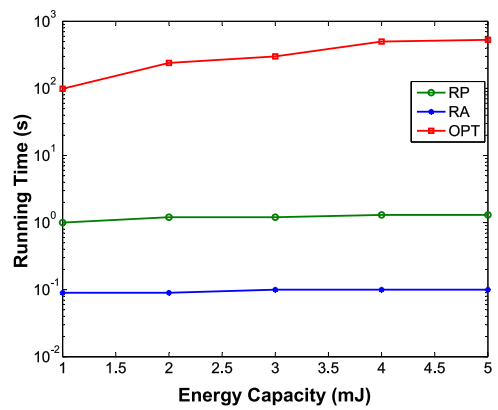

(d) Running time vs Number of chargers

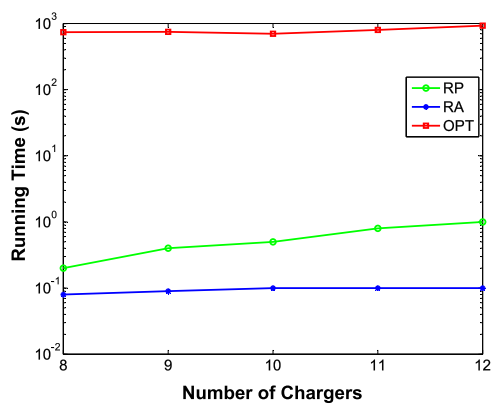

(f) Running time vs Energy Capacity.

Fig. 10. Simulation results of RoundPick.

In Figure 11, we can see that the proposed algorithm WeightGreedyPick outperforms the RRP algorithm, and the total charging periods decreases as the number of chargers grows. The total charging time grows with the growth of the number of sensors and with the growth of the capacitance.

To show the influence of step value of phase, we construct an experiment, whose result is shown in Figure 11(d). We can see that when the step value of a phase increases, the charging period also increases. This is obvious, because with a smaller step value, the change of plus areas and minus areas becomes more detailed, which may increase the possibility of better solutions.

We also construct an experiment to show the influence of the length of the charging period. We can see from Figure 12 that the total charging time grows with the growth in the length of charging period. This is obvious, because some sensors may be fully charged at the beginning of a charging period, and with longer length of the period, more energy could be wasted. Shorter length of the 


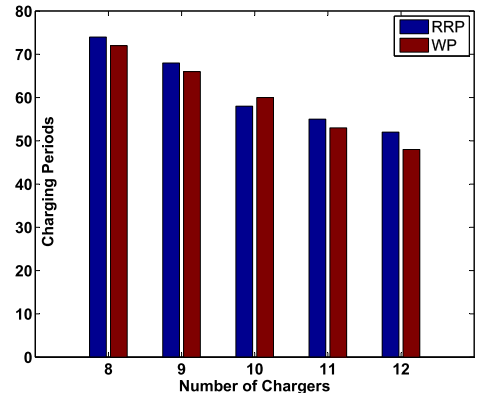

(a) Charging periods vs Number of chargers.

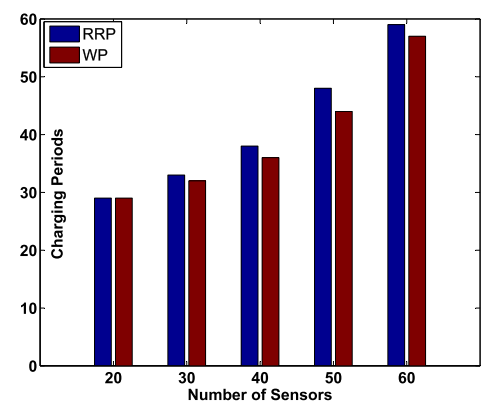

(c) Charging periods vs Number of sensors.

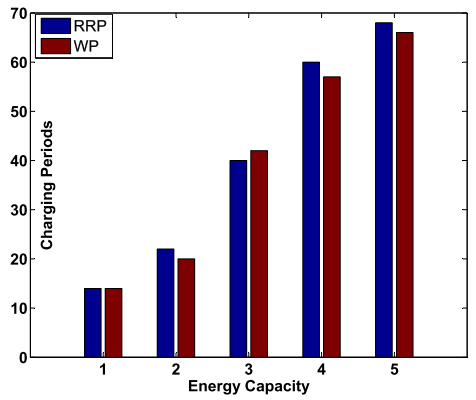

(b) Charging periods vs Energy capacity.

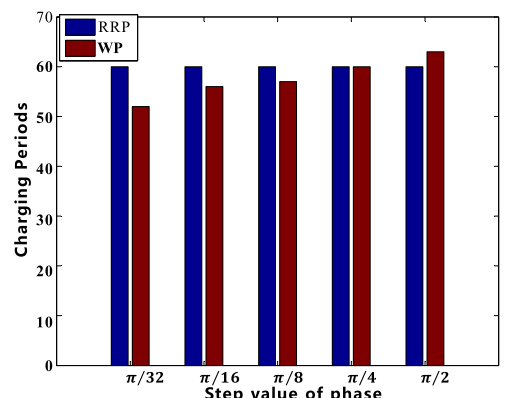

(d) Charging periods vs Step value of phase.

Fig. 11. Simulation Results of WeightGreedyPick.

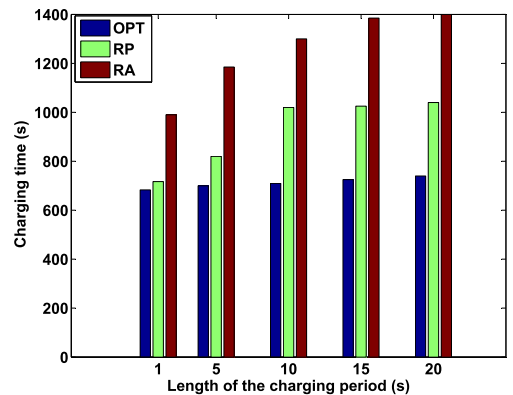

Fig. 12. Charging Time vs. The length of charging period.

period means more efficient scheduling, which gives us a better understanding of energy status of sensors.

In summary, the proposed algorithm RoundPick performs very similarly to GA (which is considered as OPT) in sparse networks and outperforms the random algorithm, and the proposed algorithm WeightGreedyPick performs better than RRP.

\section{CONCLUSION}

In this article, we study the FCS problem, addressing the nonlinear superposition charging effect caused by radio interference. We prove that this problem is NP-complete by reduction from the knapsack problem. To solve this problem, we first propose the 2-approximation algorithm FastPick 
in a $1 \mathrm{D}$ line. In a 2D plane, we discuss the special case of FCS, which we call the FCSF problem, and propose the RoundPick algorithm to solve FCSF and obtain a bound of $6-4 \epsilon$ when sensors and chargers are uniformly distributed, and $\epsilon$ is the error related to the actual charging condition. After that, we propose the WeightGreedyPick algorithm to solve FCS in a 2D plane. The simulation results show that the RoundPick can achieve a good performance that is close to that of OPT in a sparse network.

\section{REFERENCES}

A. Alphonsa and G. Ravi. 2016. Earthquake early warning system by IOT using Wireless sensor networks. In Proceedings of the International Conference on Wireless Communications, Signal Processing and Networking (WiSPNET'16). IEEE, 12011205.

Haipeng Dai, Yunhuai Liu, Guihai Chen, Xiaobing Wu, and Tian He. 2014. SCAPE: Safe charging with adjustable power. In Proceedings of the 2014 IEEE 34th International Conference onDistributed Computing Systems (ICDCS'14). IEEE, 439-448.

Haipeng Dai, Yunhuai Liu, Alex X. Liu, Lingtao Kong, Guihai Chen, and Tian He. 2016. Radiation constrained wireless charger placement. In Proceedings of the 35th Annual IEEE International Conference on Computer Communications (INFOCOM'16). IEEE, 1-9.

Erik D. Demaine, Uriel Feige, MohammadTaghi Hajiaghayi, and Mohammad R. Salavatipour. 2008. Combination can be hard: Approximability of the unique coverage problem. SIAM f. Comput. 38, 4 (2008), 1464-1483.

Chen Deng-Peng. 2009. Method and apparatus for optical wireless charging. US Patent 7,514,899.

Zhi Ang Eu, Winston K. G. Seah, and Hwee-Pink Tan. 2008. A study of MAC schemes for wireless sensor networks powered by ambient energy harvesting. In Proceedings of the 4th Annual International Conference on Wireless Internet. ICST, 78.

Xenofon Fafoutis and Nicola Dragoni. 2011. ODMAC: An on-demand MAC protocol for energy harvesting-wireless sensor networks. In Proceedings of the 8th ACM Symposium on Performance Evaluation of Wireless ad Hoc, Sensor, and Ubiquitous Networks. ACM, 49-56.

Arnaud Fréville. 2004. The multidimensional 0-1 knapsack problem: An overview. Eur. f. Operat. Res. 155, 1 (2004), 1-21.

Om P. Gandhi, L. Lloyd Morgan, Alvaro Augusto de Salles, Yueh-Ying Han, Ronald B. Herberman, and Devra Lee Davis. 2012. Exposure limits: The underestimation of absorbed cell phone radiation, especially in children. Electromagn. Biol. Med. 31, 1 (2012), 34-51.

Yuan Gao, Cong Wang, and Yuanyuan Yang. 2015. Joint wireless charging and sensor activity management in wireless rechargeable sensor networks. In 2015 44th International Conference on Parallel Processing (ICPP'15). IEEE, 789-798.

Jorge L. Garcia, Annamarie Burke, B. Juan, Charles B. Swope, and Joseph Patino. 1999. Wireless battery charging system having adaptive parameter sensing. US Patent 5,963,012.

Peng Guo, Xuefeng Liu, Shaojie Tang, and Jiannong Cao. 2016. Concurrently wireless charging sensor networks with efficient scheduling. IEEE Trans. Mobile Comput. 16, 9 (2016), 2450-2463.

Shibo He, Jiming Chen, Fachang Jiang, David K. Y. Yau, Guoliang Xing, and Youxian Sun. 2013. Energy provisioning in wireless rechargeable sensor networks. IEEE Trans. Mobile Comput. 12, 10 (2013), 1931-1942.

Jason Hsu, Sadaf Zahedi, Aman Kansal, Mani Srivastava, and Vijay Raghunathan. 2006. Adaptive duty cycling for energy harvesting systems. In Proceedings of the 2006 International Symposium on Low Power Electronics and Design. ACM, 180-185.

S Jegadeesan and GKD Prasanna Venkatesan. 2016. Distant biometry in cattle farm using wireless sensor networks. In Proceedings of the International Conference on Communication and Electronics Systems (ICCES'16). IEEE, 1-5.

Xiaofan Jiang, Joseph Polastre, and David Culler. 2005. Perpetual environmentally powered sensor networks. In Proceedings of the 4th International Symposium on Information Processing in Sensor Networks. IEEE Press, 65.

Aman Kansal, Jason Hsu, Sadaf Zahedi, and Mani B. Srivastava. 2007. Power management in energy harvesting sensor networks. ACM Trans. Embed. Comput. Syst. 6, 4 (2007), 32.

Zi Li, Yang Peng, Daji Qiao, and Wensheng Zhang. 2014. Joint charging and rate allocation for utility maximization in sustainable sensor networks. In Proceedings of the 2014 11th Annual IEEE International Conference on Sensing, Communication, and Networking (SECON'14). IEEE, 459-467.

Peng Lin, Chunming Qiao, and Xin Wang. 2004. Medium access control with a dynamic duty cycle for sensor networks. In Proceedings of the 2004 IEEE Wireless Communications and Networking Conference, Vol. 3. IEEE, 1534-1539.

Zhi Ma, Jie Wu, Sheng Zhang, and Sanglu Lu. 2018a. Fast interference-aware scheduling of multiple wireless chargers. In Proceedings of the 2018 IEEE 15th International Conference on Mobile Ad Hoc and Sensor Systems (MASS'18). IEEE, 344-352.

Zhi Ma, Jie Wu, Sheng Zhang, and Sanglu Lu. 2018b. Prolonging WSN lifetime with an actual charging model. In Proceedings of the 2018 IEEE Wireless Communications and Networking Conference (WCNC'18). IEEE, 1-6. 
Silvano Martello, David Pisinger, and Paolo Toth. 2000. New trends in exact algorithms for the 0-1 knapsack problem. Eur. f. Operat. Res. 123, 2 (2000), 325-332.

M. Yousof Naderi, Kaushik R. Chowdhury, and Stefano Basagni. 2015. Wireless sensor networks with RF energy harvesting: Energy models and analysis. In Proceedings of the 2015 IEEE Wireless Communications and Networking Conference (WCNC'15). IEEE, 1494-1499.

M. Yousof Naderi, Kaushik R. Chowdhury, Stefano Basagni, Wendi Heinzelman, Swades De, and Soumya Jana. 2014a. Experimental study of concurrent data and wireless energy transfer for sensor networks. In Proceedings of the 2014 IEEE Global Communications Conference (GLOBECOM'14). IEEE, 2543-2549.

M. Yousof Naderi, Prusayon Nintanavongsa, and Kaushik R. Chowdhury. 2014b. RF-MAC: A medium access control protocol for re-chargeable sensor networks powered by wireless energy harvesting. IEEE Trans. Wireless Commun. 13, 7 (2014), 3926-3937.

Yuki Nishikawa, Takuya Sasamura, Yoichi Ishizuka, Satoshi Sugimoto, Shohei Iwasaki, Hongyan Wang, Tomoyuki Fujishima, Takafumi Fujimoto, Koichiro Yamashita, Takahisa Suzuki, et al. 2018. Design of stable wireless sensor network for slope monitoring. In Proceedings of the 2018 IEEE Topical Conference on Wireless Sensors and Sensor Networks (WiSNet'18). IEEE, 8-11.

Dusit Niyato, Ekram Hossain, Mohammad M. Rashid, and Vijay K. Bhargava. 2007. Wireless sensor networks with energy harvesting technologies: A game-theoretic approach to optimal energy management. IEEE Wireless Commun. 14, 4 (2007), 90-96.

Adam B. Noel, Abderrazak Abdaoui, Tarek Elfouly, Mohamed Hossam Ahmed, Ahmed Badawy, and Mohamed S. Shehata. 2017. Structural health monitoring using wireless sensor networks: A comprehensive survey. IEEE Communications Surveys \& Tutorials 19, 3 (2017), 1403-1423.

Yawei Pang, Zaixin Lu, Miao Pan, and Wei Wayne Li. 2014. Charging coverage for energy replenishment in wireless sensor networks. In Proceedings of the 2014 IEEE 11th International Conference on Networking, Sensing and Control (ICNSC'14). IEEE, 251-254.

Joseph A. Paradiso. 2006. Systems for human-powered mobile computing. In Proceedings of the 2006 43rd ACM/IEEE Design Automation Conference. IEEE, 645-650.

Chulsung Park and Pai H. Chou. 2006. Ambimax: Autonomous energy harvesting platform for multi-supply wireless sensor nodes. In 2006 3rd Annual IEEE Communications Society on Sensor and ad Hoc Communications and Networks, Vol. 1. IEEE, 168-177.

Yang Peng, Zi Li, Wensheng Zhang, and Daji Qiao. 2010. Prolonging sensor network lifetime through wireless charging. In Proceeedings of the 2010 IEEE 31st Real-time Systems Symposium (RTSS'10). IEEE, 129-139.

Vijay Raghunathan, Aman Kansal, Jason Hsu, Jonathan Friedman, and Mani Srivastava. 2005. Design considerations for solar energy harvesting wireless embedded systems. In Proceedings of the 4th International Symposium on Information Processing in Sensor Networks. IEEE Press, 64.

Fahira Sangare, Yong Xiao, Dusit Niyato, and Zhu Han. 2017. Mobile charging in wireless-powered sensor networks: Optimal scheduling and experimental implementation. IEEE Trans. Vehic. Technol. 66, 8 (2017), 7400-7410.

Winston K. G. Seah, Zhi Ang Eu, and Hwee-Pink Tan. 2009. Wireless sensor networks powered by ambient energy harvesting (WSN-HEAP)-Survey and challenges. In Proceedings of the 2009 1st International Conference on Wireless Communication, Vehicular Technology, Information Theory and Aerospace \& Electronic Systems Technology. IEEE, 1-5.

Nathan S. Shenck and Joseph A. Paradiso. 2001. Energy scavenging with shoe-mounted piezoelectrics. IEEE Micro 3 (2001), 30-42.

Farhan Simjee and Pai H. Chou. 2006. Everlast: long-life, supercapacitor-operated wireless sensor node. In Proceedings of the 2006 International Symposium on Low Power Electronics and Design. ACM, 197-202.

Sujesha Sudevalayam and Purushottam Kulkarni. 2010. Energy harvesting sensor nodes: Survey and implications. IEEE Commun. Surv. Tutor. 13, 3 (2010), 443-461.

Bin Tong, Zi Li, Guiling Wang, and Wensheng Zhang. 2010. How wireless power charging technology affects sensor network deployment and routing. In Proceedings of the 2010 IEEE 30th International Conference on Distributed Computing Systems. IEEE, 438-447.

Wei Ye, John Heidemann, and Deborah Estrin. 2004. Medium access control with coordinated adaptive sleeping for wireless sensor networks. IEEE/ACM Trans. Netw. 12, 3 (2004), 493-506.

Sheng Zhang, Zhuzhong Qian, Jie Wu, Fanyu Kong, and Sanglu Lu. 2018. Wireless charger placement and power allocation for maximizing charging quality. IEEE Trans. Mobile Comput. 17, 6 (2018), 1483-1496.

Sheng Zhang, Jie Wu, and Sanglu Lu. 2015. Collaborative mobile charging. IEEE Trans. Comput. 3, 64 (2015), 654-667.

Received January 2019; revised July 2019; accepted July 2019 